\title{
SARAH : modèle de simulation de la croissance, du développement et de l'architecture des sys- tèmes racinaires
}

\author{
Loïc PAGES \& Franck ARIES (*) \\ I.N.R.A., Station d'Agronomie \\ (*) Station de Biométrie, Centre de Recherches d'Avignon, Domaine Saint-Paul, F84I40 Montfavet
}

\begin{abstract}
On présente, dans cet article, SARAH, un modèle mathématique de simulation de l'architecture tridimensionnelle des systèmes racinaires. Il s'agit d'un modèle numérique dans lequel le temps est discrétisé et déroulé par pas constants. A chaque pas de temps, les différents axes du système racinaire sont allongés et peuvent être ramifiés. Les hypothèses de base du modèle, pour la croissance et la ramification des axes, formalisent un ensemble de connaissances sur la morphogenèse des systèmes racinaires. L'allongement de chaque axe est fonction de son ordre et éventuellemnt des conditions locales de croissance de l'apex. Les ramifications sont émises de façon acropète, à une distance préalablement fixée de l'apex et des ramifications précédentes. Elles apparaissent le long des génératrices face aux pôles de xylème, avec un angle d'insertion caractéristique de leur ordre.

A partir de la structure ramifiée qui est générée par le modèle, il est possible de calculer différentes «sorties", comme par exemple : des cinétiques de croissance et développement, des profils racinaires mono-dimensionnels, des cartes d'impacts racinaires... Les simulations peuvent ainsi être confrontées aux observations. Quelques exemples illustrent les possibilités de validation de ce type de modèle.
\end{abstract}

Mots clés additionnels : Racine, distribution spatiale, morphogenèse, modèle mathématique.

SARAH : a simulation model for growth, development and architecture of root systems.

A numerical computer model (SARAH) is presented which simulates three-dimensional root system architectures at discrete time steps. At each time step, the axes of the root system experience elongation and possibly branching. Growth and branching rules are based on current knowledge about root system morphogenesis. The elongation of each axis depends on its ramification order and on local growing conditions of the apex. Branches appear acropetally at a specified distance from the apex and from former branches, along ranks facing xylem poles, with a branching angle depending on their order. From the three-dimensional branching pattern, various outputs, such as growth and development kinetics, vertical root density distributions, or cross-section maps can be computed and compared to observed data. Some examples of such possible validations are presented.

Additional key words : Root, spatial distribution, morphogenesis, mathematical model.

\section{INTRODUCTION}

Alors que le système racinaire est si important dans la vie des plantes et que celles-ci utilisent une large proportion de leurs assimilats pour le produire et le maintenir, nos connaissances sur son fonctionnement restent très limitées. Pour comprendre comment il absorbe l'eau et les éléments minéraux, il est indispensable d'étudier sa physiologie, mais aussi ses caractéristiques spatiales et structurelles.
En effet, dans le sol où les ressources en eau et en éléments minéraux sont souvent dispersées à faible concentration, ou réparties de façon hétérogène, la distribution spatiale des racines va conditionner le niveau d'absorption, et ceci d'autant plus que la mobilité des substances à prélever sera plus faible (TAYLOR \& KLEPPER, 1978).

Pour décrire la morphologie des systèmes racinaires, nous pouvons distinguer 2 types d'approche : d'une part des analyses de la variation des densités racinaires dans 
le sol (exprimées par exemple en longueur de racines par unité de volume de sol), d’autre part des études de la structure du système racinaire.

Le premier type d'approches aboutit à la réalisalion de profils racinaires (distribution des densités suivart la verticale) ou de cartes bi-dimensionnelles (BöııM. 1979), parfois mises en relation avec les cartes de caractéristiques du sol (TARDIEU \& MANICHON, 1986). Ces descriptions, généralement associées à des méthodes d'observation destructives (carottes de sol, tranchées), présentent généralement un constat de l'état de l'enracinement, pour un diagnostic de fonctionnement à un moment donné. Quelques modèles dynamiques monodimensionnels générant au cours du temps des cartes de densité racinaire ont été réalisés (PAGE \& GERWITZ, 1974 ; HUCK \& HiLLEL, 1983) mais ils ne peuvent bien sûr prendre en compte les hétérogénéités du sol (et par conséquent de la distribution racinaire) dans le plan horizontal.

Les études de structure du système racinaire prennent en compte les liaisons entre racines : le système racinaire n'est plus envisagé comme un « nuage de densité », mais comme un arbre. Ces études introduisent une dimension morphogénétique dans la description. Les résultats en sont: des typologies de forme (JENIK, 1978; KAHN, 1977), des statistiques sur les réseaux racinaires (FITTER, 1982 ; FITTER, 1987), et des modèles de développement à formalisme plus ou moins mathématique, et s’intéressant à tout ou partie du système racinaire (HACKETT \& ROSE, 1972; LUNGLEY, 1973 ; RoSE. 1983; KLEPPER et al., 1984; PICARD et al., 1985).

Pour modéliser la morphogenèse du système racinaire, il nous apparaît important de s'appuyer sur les connaissances biologiques concernant la croissance et la ramification des racines, comme cela est fait sur les parties aériennes des arbres (DE REFFYE el al., 1986). Pour être utilisable par l'agronome, ces connaissances doivent être assemblées et formalisées à travers un modèle mathématique qui permettra de faire des simulations et d'avoir donc des sorties quantitatives permettant d'une part de tester des hypothèses de croissance et développement, et d'autre part de fournir des entrées pour les modèles d'absorption.

Précédemment, HACKETT \& ROSE (1972), puis ROSE (1983) ont construit des modèles algébriques fondés sur les caractéristiques de croissance et de ramification des racines, mais ces modèles ne se préoccupent que des aspects cinétiques du problème, et non de ses aspects géométriques. D'autre part, leur formalisation implique la constance des vitesses de croissance et de ramification dans le temps et dans l'espace, ce qui peut être considérablement restrictif. LUNGLEY (1973) s'abstrait de cette contrainte grâce à un modèle numérique, et il prend en compte une partie de la géométrie du système, ce qui lui permet de générer des profils racinaires.

Nous poursuivons cette démarche en proposant ici un modèle d'architecture dynamique et tri-dimensionnel. Contrairement aux précédents essais cités, nous nous efforçons d'aborder véritablement les problèmes spatiaux, qui sont fondamentaux vis-à-vis du fonctionnement.

Le but de cet article est de présenter le modèle comme une méthode d’étude du système racinaire

Dans une première partie, nous présenterons la structure générale du modèle, ainsi que les hypothèses de base et les paramètres quantitatifs à estimer pour son étalonnage. Nous verrons à ce titre que les hypothèses de base sont une formulation explicite et une traduction mathématique d'un ensemble de règles morphogénétiques couramment observées par les biologistes.

Nous discuterons ensuite quelques exemples de sorties du modèle permettant sa validation et offrant les entrées i d'autres modèles de fonctionnement, étudiant notamment l'absorption hydrique et/ou minérale. L'estimation des paramètres ainsi que la validation seront approfondis sur des cas précis dans un prochain article.

\section{HYPOTHÈSES DE BASE DU MODÈLE}

\section{A. Définitions et caractéristiques générales}

Le système racinaire est une structure ramifiée : un arbre au sens mathématique. Nous appellerons «axe» ou " racine " l'entité générée par un seul méristème. Ces axes peuvent être hiérarchisés par leur "ordre »: les racines d'ordres $\mathrm{n}$ portent des ramifications d'ordre $\mathrm{n}+1$.

CANNON (1949) \& KAHN (1977) distinguent 2 types principaux de systèmes racinaires :

- Le système racinaire primaire possède un axe racinaire orthotrope (que nous appellerons arbitrairement d'ordre 1) qui élabore un système plagiotrope par ramification (axes d'ordre 2, puis 3, etc...). On parle dans ce cas d'un système " pivotant".

- Le système racinaire secondaire est émis par la base de la tige, qui joue le rôle de l'ordre 1, et donne naissance à des racines adventives (arbitrairement d'ordre 2) qui se ramifient également. On parle alors de système « fasciculé ».

Chez certaines espèces (maïs par exemple), ces 2 types de systèmes racinaires peuvent coexister; on obtient un système racinaire mixte.

Le modèle simule la génération d'une structure tridimensionnelle au cours du temps. Pour ce faire, celui-ci est discrétisé, et déroulé par pas constants. Il est défini par l"utilisateur et peut correspondre au temps astronomique, à des sommes de températures (degré-jour), ou à un temps biologique (phyllochrone). La durée totale de la simulation et le pas de discrétisation sont également spécifiés par l'utilisateur. Au fur ct à mesure que la structure est génćrée, elle est stockée dans un fichier qui est mis à jour à chaque pas de temps. Sur ce fichier, chaque enregistrement est un "segment racinaire», c'est-à-dire un morceau d'axe généré pendant un pas de temps. La génération de la structure se traduit par un traitement de chacun des axes à chaque pas de temps avec 2 opérations fondamentales : croissance et ramification que nous allons détailler.

Notons au préalable que, aussi bien pour la croissance que pour la ramification, les "parametres » que nous allons présenter sont en fait, dans le programme, des fonctions qui sont évaluées à chaque appel au cours du déroulement de la simulation. Ainsi, ces paramètres de base peuvent avoir une valeur constante durant toute la simulation (la fonction concernée renvoie alors une valcur constante) ou dépendre du temps, de l'espace, de la position dans la structure, etc... 
Notons également que dans le modèle, les différents ordres sont tous traités avec les mêmes hypothèses de base, seules les valeurs des paramètres changent de l'un à l'autre.

\section{B. Croissance des axes}

Pour représenter l'allongement d'un axe dans l'espace à 3 dimensions pendant un pas de temps, on doit se préoccuper de la longueur de cet accroissement (que nous appellerons "Elong»), et de sa direction. Ces caractéristiques sont représentées par un vecteur d'accroissement.

La longueur d'accroissement (Elong) est calculée à chaque pas de temps et pour chaque axe par une fonction qui reçoit comme entrées: l'ordre de l'axe considéré, son âge, la localisation $(\mathrm{x}, \mathrm{y}, \mathrm{z})$ de son apex. Ainsi, l'utilisateur choisit le sous-modèle qui décrit l'accroissement. Il pourra par exemple supposer que la vitesse de croissance d'un axe est constante et ne dépend que de l'ordre, et dans ce cas, il devra seulement estimer un paramètre par ordre décrit qui sera sa vitesse de croissance. Mais cette fonction permet également à l'utilisateur d'intégrer un sous-modèle plus complexe qui définit l'allongement d'un axe en fonction de sa position dans la structure (ordre, âge) et/ou en fonction des propriétés de l'environnement local de l'apex (température, impédance mécanique). L'utilisateur peut également simuler la mortalité d'un axe à un moment donné (Elong $=0$ à partir de ce moment).

La direction de croissance est également calculée par un module chargé de décrire les tropismes (géotropisme notamment) et les réactions aux contraintes mécaniques (contournements d'obstacles par exemple). Dans les exemples qui suivent, le modèle simule uniquement la courbure géotropique des racines. Le vecteur qui oriente le segment d'accroissement à un pas de temps donné est égal à la somme de 2 vecteurs qui vont s'ajouter :

- un vecteur de norme 1 ayant la direction de la croissance au pas de temps précédent (la direction d'émission d'un axe d'ordre 1 doit être définie spécifiquement, et celle des axes d'ordre supérieur est définie par la procédure de ramification);

- un vecteur " géotropisme » $(\overrightarrow{\mathrm{g}})$ vertical orienté vers le bas, de norme proportionnelle à l'allongement pendant le pas de temps considéré, et proportionnelle à un paramètre Géo fixé par l'utilisateur pour chaque ordre $(\|\vec{g}\|=$ Elong-Géo).

\section{Ramification des axes}

\section{Localisation de la ramification}

Les hypothèses concernant la localisation des racines latérales reprennent les observations faites par d'autres auteurs sur différentes espèces, aussi bien sur céréales (HACKETT \& ROSE, 1972; LunGley, 1973), que sur arbres (LYFORD, 1980). Ainsi, la racine peut être découpée en 3 zones (fig. 1): une zone basale sans ramification, une zone intermédiaire où les ramifications sont distribuées relativement régulièrement, et une zone apicale sans ramification. L'émission de racines latérales se fait à l'interface de ces 2 dernières zones. Au cours de l'allongement de l'axe, la zone ramifiée s'étend en suivant la progression de l'apex (McCully, 1975).

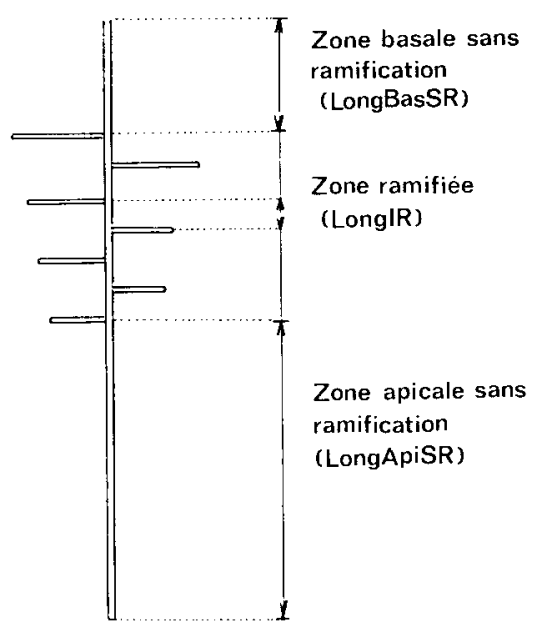

Figure 1

Description des différentes zones sur une racine, et paramètres associés. Description of the different zones on a root and associated parameters.

Dans le modèle, nous supposerons qu'un axe ne peut se ramifier que quand il a atteint une longueur supérieure à la somme entre la longueur de la zone basale non ramifiée (paramètre «LongBasSR ») et la longueur de la zone apicale non ramifiée (paramètre «Long $A$ $p i S R »)$. Les ramifications suivantes apparaissent de façon strictement acropète, séparées les unes des autres par une certaine distance inter-ramification (paramètre «LongIR»), et à une distance de l'apex toujours supérieure au paramètre «LongApiSR».

Finalement :

- la première ramification apparaît sur un axe dès que sa longueur est supérieure à la somme «LongBasSR + Long ApiSR»;

- les ramifications suivantes apparaissent tant que la distance entre l'apex et la dernière ramification émise (la plus proche de celui-ci) est supérieure à la somme "LongIR + LongApiSR»;

- lorsqu'une ramification est émise, elle est située à la distance "LongIR» de la précédente, ou à une distance "LongBasSR » de la base s’il s'agit de la première ramification.

La vision que nous présentons ici est simplificatrice : d'après GEISSBUhLER, 1953 (in McCULLY, 1975) la longueur de la zone apicale non ramifiée est corrélée à la vitesse de croissance de la racine. De plus, cette modélisation n'est proche de la réalité que lorsque la racine pousse sans trop de perturbations, dans un milieu relativement homogène. Les paramètres définis sont alors stables, et traduisent un ensemble de corrélations morphogénétiques. Mais dans le cas où la croissance d'un pivot est inhibée par exemple, ces paramètres de localisation des ramifications sont profondément modifiés (PILET, 1973; RIEDACKER \& BELGRAND, 1983). Par ailleurs, HACKETT (1972) montre que la distance entre ramifications peut être modifiée localement sur un système racinaire par un apport d'azote $\left(\mathrm{NO}_{3}^{-}\right)$. 
Ces considérations justifient le fait que, dans notre modèle, les paramètres de la ramification puissent être des fonctions du temps et de l'espace.

\section{Orientation de la ramification (fig. 2)}

Le départ d'un axe doit également être orienté dans l'espace. Nous caractérisons cette orientation par 2 angles : l'angle d'insertion (AngIns) et l'angle de génératrice (AngGen).
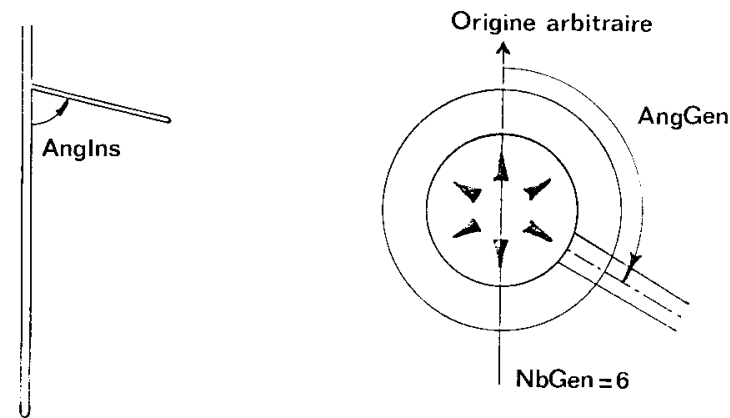

Figure 2

Orientation de la ramification avec les deux angles de caractérisation. Branch orientation with the two characteristic angles.

L'angle d'insertion est défini comme l'angle entre l'axe mère et sa ramification.

Dans le sens radial, l'émergence des racines latérales se fait suivant un schéma généralement précis, soit juste en face des pôles de protoxylème pour une majorité d'espèces, soit entre ces pôles chez quelques familles. Il en résulte des alignements des racines latérales suivant les génératrices d'un axe (McCULLY, 1975). De la mème façon que sur les parties aériennes, il y a donc sur un axe racinaire un nombre limité de directions d'émergence des ramifications. Le modèle suppose que les génératrices sont régulièrement réparties autour de l'axe, et qu'il suffit donc d'en connaitre le nombre (NbGen) pour en déduire les différentes orientations, ou angles (AngGen).

Cependant, on ne rencontre pas sur les racines une «rhizotaxie» qui serait l'analogue de la phyllotaxie caulinaire. Dans le modèle, à chaque ramification émise, le rang de la génératrice d'origine est tiré au hasard, et l'angle d'orientation radiale automatiquement déduit.

AnGen $=2 \pi N / N b G e n$ ( $\mathrm{N}$ étant un entier tiré au hasard entre 1 et $N b G e n$ inclus, pour chaque racine émise).

En fait, divers auteurs observent que la séquence d'initiation des latérales entre les génératrices n'est pas totalement aléatoire (RIOPEL, 1966; RIOPEL, 1969; Charlton, 1983). Selon Charlton (1983) qui a étudié plusieurs espèces, une racine latérale déjà initiée sur un axe va tendre à inhiber l'émergence d'une autre latérale à sa proximité sur la même génératrice; par contre, il n'y aurait pas de corrélations entre génératrices différentes. Par ailleurs, McCulLY (1975) note chez certaines espèces une tendance à l'émergence de plusieurs racines latérales du même point. Enfin, la courbure d'un axe induit l'apparition de latérales agrégées au niveau de cette courbure, et insérées du côté convexe préférentiellement. Cependant, ces aspects ne sont pas encore connus assez précisément pour permettre une modélisation plus fine.

\section{Paramètres du modèle (tabl. 1)}

L'utilisateur peut choisir le nombre d'ordres racinaires qu'il va décrire par le modèle, car ce nombre n'est pas limité par le programme. Il spécifie tout d'abord la durée de simulation ainsi que la valeur du pas de temps. S'il veut faire une simulation de l'architecture d'un système racinaire, il doit définir pour chaque ordre:

- l'élongation (Elong);

- les longueurs : de la zone apicale sans ramification (LongApiSr), de la zone basale sans ramification (LongBasSR), et inter-ramification (LongIR);

- l'angle d'insertion (AngIns);

- le nombre de génératrices (NbGen);

- le coefficient de géotropisme (Géo).

Ces 3 derniers paramètres (AngIns, NbGen, Géo) ne sont à fournir que si l'on veut représenter le système racinaire dans l'espace.

Grâce à la structure du programme associé au modèle, il est très facile de transformer ces paramètres (constantes définies pour chaque ordre) en fonction du temps et de l'espace. L'utilisateur doit alors définir ces fonctions dans le programme et fournir les paramètres utiles. Cette souplesse permet en particulier de faire une partition de l'espace en différentes zones induisant chacune des caractéristiques de croissance et de ramification propres, qui proviendraient par exemple des variations spatiales et temporelles de température, d'impédance mécanique, etc...

Il est également possible de sortir de ce cadre fortement déterministe, et de tirer au hasard certaines de ces valeurs (Elong, AngIns, par exemple) dans des distributions connues. Le modèle stochastique permet alors des études de variabilité de caractéristiques induites.

\section{DISCUSSION SUR DES EXEMPLES DE SORTIES}

L'estimation des paramètres et la validation de ce modèle posent bien sûr les problèmes liés aux difficultés

TABLEAU 1

Récapitulatif des noms de paramètres utilisés par le modèle, avec les unités correspondantes.

Parameter names used in the model, with corresponding units.

\begin{tabular}{|c|c|c|}
\hline Symbole & Signification & Dimension \\
\hline Elong & $\begin{array}{l}\text { Elongation de l'axe } \\
\text { pendant un pas de temps }\end{array}$ & Longueur/temps \\
\hline LongApiSR & $\begin{array}{l}\text { Longueur de la zone apicale } \\
\text { sans ramification }\end{array}$ & Longueur \\
\hline LongBasSR & $\begin{array}{l}\text { Longueur de la zone basale } \\
\text { sans ramification }\end{array}$ & Longueur \\
\hline LongIR & $\begin{array}{l}\text { Distance entre deux } \\
\text { ramifications successives }\end{array}$ & Longueur \\
\hline AngIns & Angle d'insertion & Angle \\
\hline NbGen & Nombre de génératrices & - \\
\hline Géo & Géotropisme & - \\
\hline
\end{tabular}


TABLEAU 2

Valeurs des paramètres utilisés dans les 4 simulations (figures $3,5,6)$ indicées $A, B, C$ et $D$. La durée de simulation est de 30 jours.

Parameter values used in the 4 simulations (figures 3, 5, 6) labelled A, B, C and D. The simulation duration is 30 days.

\begin{tabular}{|c|c|c|c|c|c|c|c|c|c|c|c|c|}
\hline \multirow[b]{2}{*}{ Ordre } & \multicolumn{3}{|c|}{ Simulation A } & \multicolumn{3}{|c|}{ Simulation B } & \multicolumn{3}{|c|}{ Simulation C } & \multicolumn{3}{|c|}{ Simulation D } \\
\hline & 1 & 2 & 3 & 1 & 2 & 3 & 1 & 2 & 3 & 1 & 2 & 3 \\
\hline Elong (cm/jour) & 5 & 3 & 0,5 & 4 & 4 & 0,3 & 0,07 & 5 & 0,3 & 5 & $6 * \mathrm{e}^{0.3 * \hat{\mathrm{agc}}}$ & 0.2 \\
\hline LongApiSR $(\mathrm{cm})$ & 25 & 20 & - & 40 & 5 & - & 0 & 5 & - & 10 & 5 & - \\
\hline LongBasSR $(\mathrm{cm})$ & 5 & 4 & - & 6 & 2 & - & 0,5 & 10 & - & 5 & 3 & - \\
\hline LongIR $(\mathrm{cm})$ & 3 & 4 & - & 2 & 4 & - & 0,1 & 2 & - & 3 & 3 & - \\
\hline NbGen & 6 & 4 & - & 9 & 5 & - & 17 & 6 & - & 7 & 5 & - \\
\hline AngIns (rad) & - & 1,5 & 1,4 & - & 1,57 & 1,3 & - & 1,1 & 1,2 & - & 1,5 & 1,5 \\
\hline Géo & 0,1 & 0,02 & 0 & 0,1 & 0 & 0 & 0,1 & 0,05 & 0,03 & 0,1 & 0,02 & 0 \\
\hline
\end{tabular}

La variable «âge » est l’âge du méristème de l'axe considéré.

d'observation des systèmes racinaires (BöHM, 1979). Si le suivi de la croissance et du développement est relativement aisé pour de jeunes plantes élevées en conteneurs sur substrats simples, il reste difficile à réaliser in situ.

Le modèle présenté décrit à la fois la cinétique de croissance et de développement, la structure et la disposition spatiale du système racinaire. Il peut donc être utilisé pour diverses études de croissance, d'architecture, d'absorption,... Suivant le type d'étude, les critères permettant de juger de la qualité du modèle ne seront pas les mêmes. Il revient à l'utilisateur de choisir les critères les plus pertinents dans chaque cas.

Nous donnerons ici quelques exemples de sorties du modèle pour en illustrer la richesse et discuter les possibilités de validation à partir de données classiquement recueillies sur les racines. La validation sur des cas précis sera abordée dans un prochain article.

\section{A. Projections de structures simulées (tabl. 2 et fig. 3)}

Même s'il est difficile de voir les systèmes racinaires, différents auteurs ont essayé de les représenter sous forme de croquis ou de photos, à partir d'observations faites après excavation (KUTSCHERA, 1960) ou en rhizotron. Sur ces bases morphologiques, des classifications ont été réalisées (CANNON, 1949; WEAVER, 1958; KAHN, 1977).

Un programme interactif de visualisation des systèmes racinaires simulés permet de faire des projections de ces structures tri-dimensionnelles sur divers plans choisis par l'utilisateur. La figure 3 montre quelques projections de structures obtenues en faisant varier les paramètres du modèle (tabl. 2). Ces formes peuvent être rapprochées des types de systèmes racinaires définis par CANNON (1949) sur des bases morphologiques et écologiques.

Bien que la validation visuelle soit subjective et non quantifiée, la comparaison des formes a le mérite d'être aisée, et souvent très discriminante par rapport à des critères quantitatifs globaux.

\section{B. Sorties concernant la chronologie du développement} (tabl. 3 et fig. 4)

La validation peut être faite en comparant des cinétiques d'apparition et de croissance de divers types d'axes. Ce genre d'étude a été réalisé sur racines séminales de céréales ayant poussé en solution nutritive ou en pots sur substrats simples pendant 20 à 25 jours (HACKeTt \& ROSE, 1972; Rose, 1983). Pour obtenir les données, HACKETT \& Rose (1972) partent d'un lot de plantes d'orge mises en croissance à une certaine date, et ils en récoltent périodiquement un certain nombre sur lesquelles ils mesurent les longueurs de tous les axes.
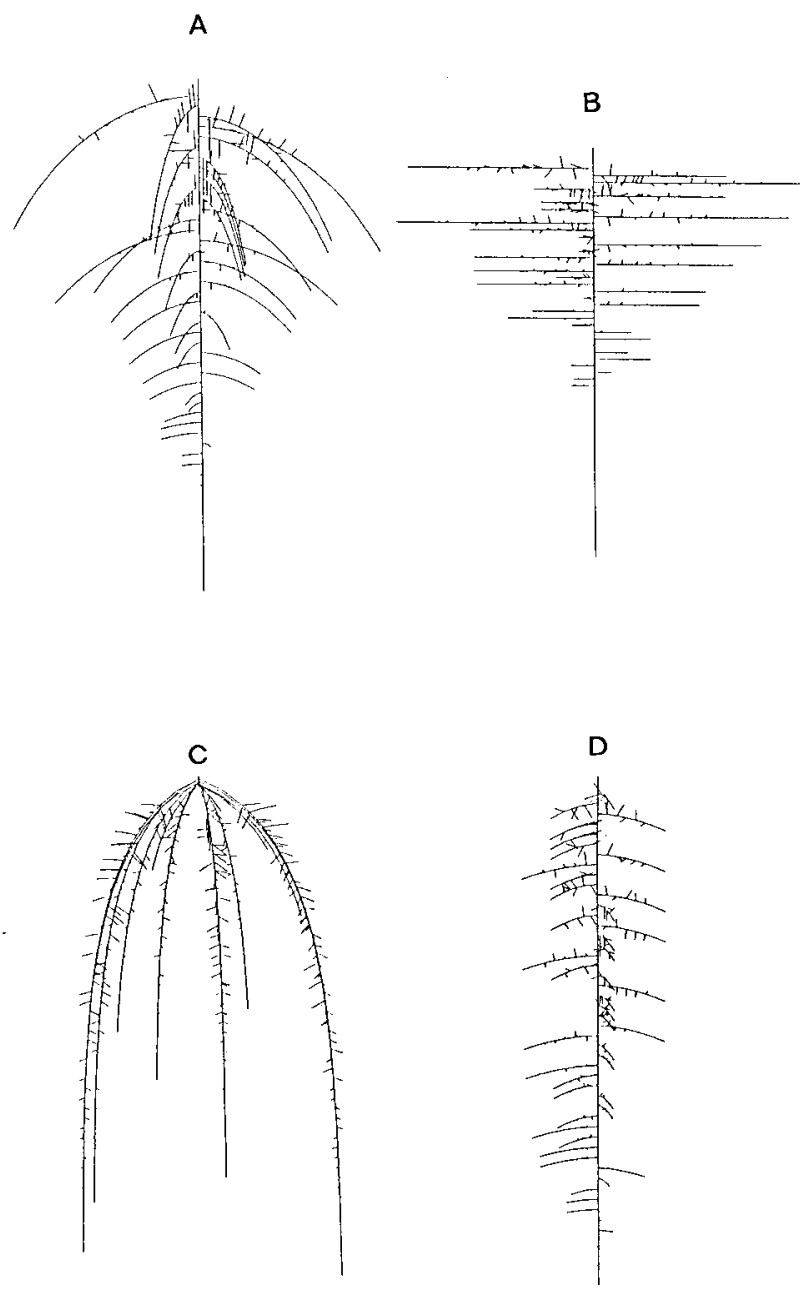

Figure 3

Projections de quelques structures tri-dimensionnelles simulées en faisant varier les paramètres (cf. tableau 2).

Projections of various three-dimensional simulated patterns, using parameters of table 2. 
Reprenant les paramètres donnés par les auteurs (tabl. 3 ), nous les avons introduits dans notre modèle. La figure 4 montre, à titre d'exemple, les résultats obtenus par simulation et la comparaison avec les expérimentations.
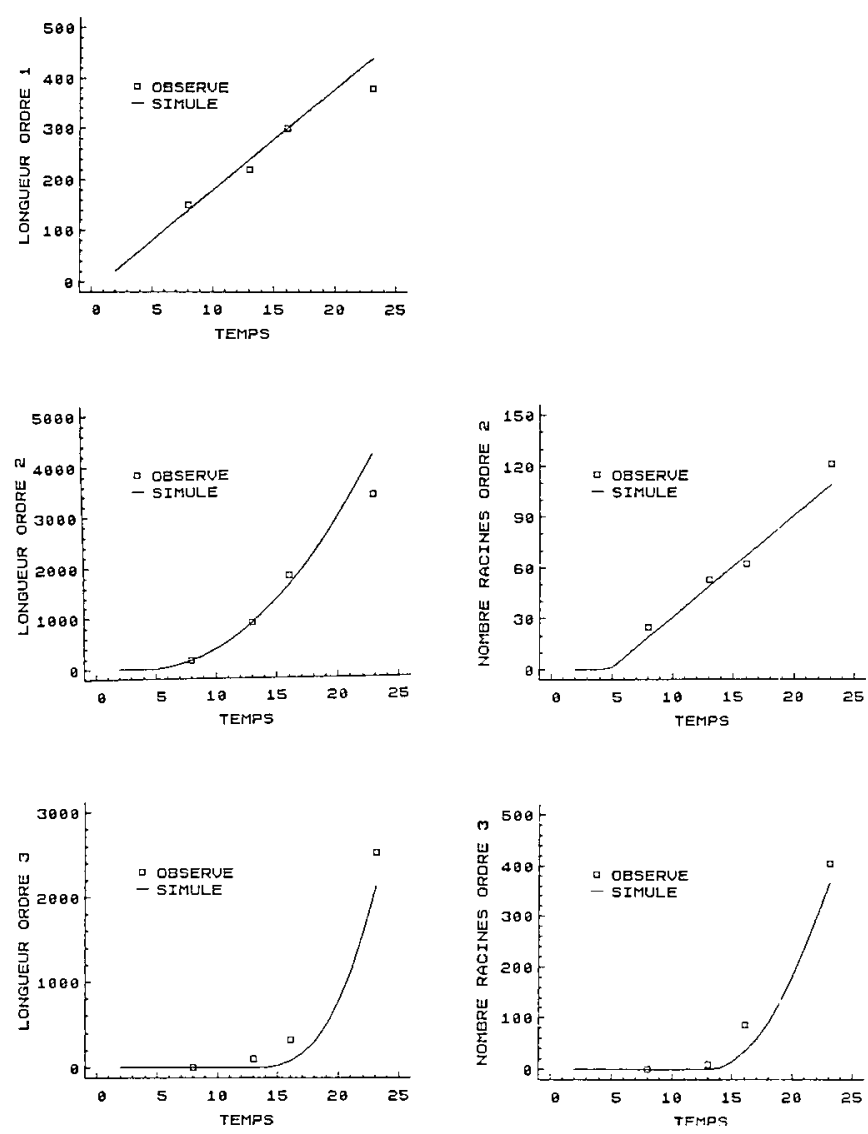

Figure 4

Cinétiques de croissance et développement, valeurs observées ( HACKETT \& ROSE, 1972) et simulees par le modele (cf. tableau 3).

Growth and development kinetics: observed values (HACKETT \& RosE 1972) and simulated values (table 3).

Du fait de difficultés pratiques (lourdeur des mesures, difficultés pour éliminer le substrat sans endommager: les racines) cette validation à partir d'une description exhaustive du système racinaire ne semble réalisable que sur des individus jeunes, élevés sur substrats artificiels.

TABLEAU 3

Valeurs des parametres estimées sur orge (d'après les travaux de HACKETT \& ROSE, 1972).

Parameter values estimated on barle. (after HACKLTT \& ROSE, 1972).

\begin{tabular}{|c|c|c|c|}
\hline $\begin{array}{l}\text { Croissance } \\
\text { (Elong) }\end{array}$ & $\begin{array}{l}\text { Longueur basale } \\
\text { sans ramif. } \\
\text { (LongBasSR) }\end{array}$ & $\begin{array}{c}\text { Distance } \\
\text { inter-ramif. } \\
\text { (LongIR) }\end{array}$ & $\begin{array}{l}\text { Longucur apicale } \\
\text { sans ramif. } \\
\text { LongApiSR) }\end{array}$ \\
\hline $\begin{array}{cc}\text { Ordre } & \text { Linéaire } \\
1 & 20 \mathrm{~mm} / \text { jour }\end{array}$ & $\begin{array}{l}\text { Constante } \\
0 \mathrm{~mm}\end{array}$ & $\begin{array}{c}\text { Constante } \\
3,3 \mathrm{~mm}\end{array}$ & $\begin{array}{l}\text { Constante } \\
60 \mathrm{~mm}\end{array}$ \\
\hline $\begin{array}{cc}\text { Ordre } & \text { Linéaire } \\
2 & 4 \mathrm{~mm} / \text { jour }\end{array}$ & $\begin{array}{l}\text { Constante } \\
0 \mathrm{~mm}\end{array}$ & $\begin{array}{l}\text { Constante } \\
3,3 \mathrm{~mm}\end{array}$ & $\begin{array}{l}\text { Constante } \\
32 \mathrm{~mm}\end{array}$ \\
\hline $\begin{array}{c}\text { Ordre Linéaire } \\
3 \quad 1,5 \mathrm{~mm} \text { jour }\end{array}$ & - & - & - \\
\hline
\end{tabular}

Sur des plants plus âgés, une telle validation peut être envisagée en pratiquant un échantillonnage dans le système racinaire, et/ou en ne considérant que les premiers ordres racinaires (le nombre d'ordres racinaires observables va dépendre de la plante étudiée)

Néanmoins, ce genre de validation paraît actuellement difficile à réaliser au champ.

\section{Profils d'enracinement (tabl. 2 et fig. 5)}

Les profils d'enracinement (relations profondeurdensité racinaire) sont abondants dans la littérature (GERWITZ \& PAGE, 1974) car ils constituent une représentation naturelle des données obtenues en prélevant des carottes de sol. La densité racinaire y est exprimée généralement par unité de volume, en longueur ou en masse, surface, nombre d'apex, etc... Nous avons réalisé un programme qui calcule les profils d'enracinement à partir des structures simulées. La figure 5 en montre quelques exemples. Ces sorties sont un outil pour étudier la sensibilité de certaines caractéristiques du profil aux variations des paramètres du modèle.
A
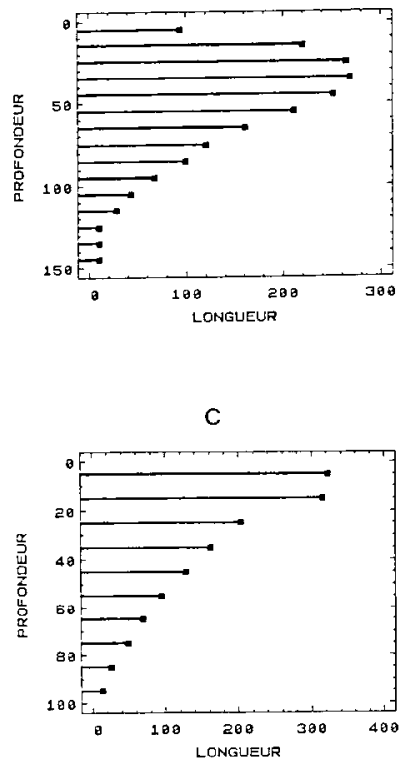

B

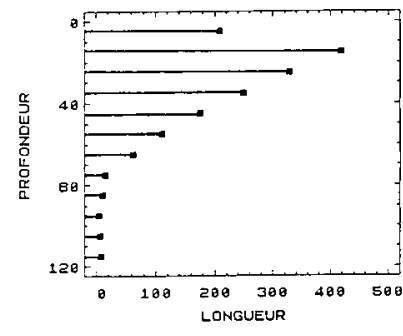

D

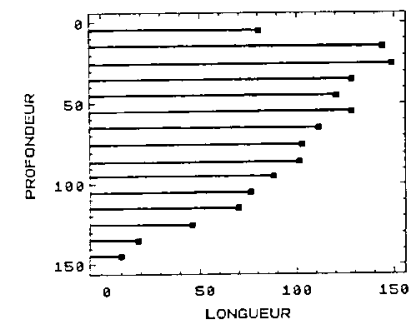

Figure 5

Profils d'enracinement simuless (ct. tableau 2). La donsité racinaire est exprimée en longueur de racines $(\mathrm{cm})$ par unité de volume ou de surface (whiti arbitraire). La profondew est expriméce con.

Simulated root profiles (table 2). Root density is expressed in root length $(\mathrm{cm})$ per soil volume or surface (arbitrary units), and depth is expressed in cm.

Cette seule description des systèmes racinaires sous forme de densité moyenne par couche de sol n'est utilisable dans un modèle d'absorption que si l'on admet l'homogénéité de la densité dans le plan horizontal, ce qui suppose une homogénéité horizontale des couches de sol, et un peuplement suffisamment dense pour que les différentes strates souterraines soient uniformément colonisées. A ce titre, notons qu'il est également possible avec un tel modèle de calculer sous différentes hypothèses (paramètres du modèle, densité du peuple- 
A1

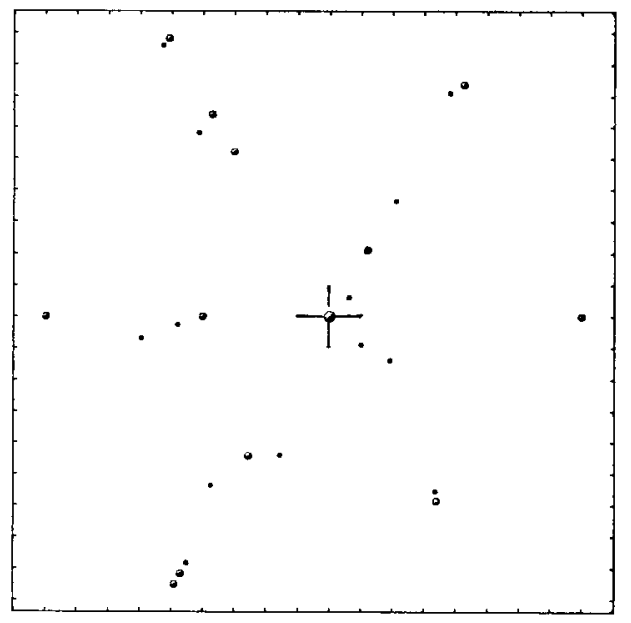

A 2

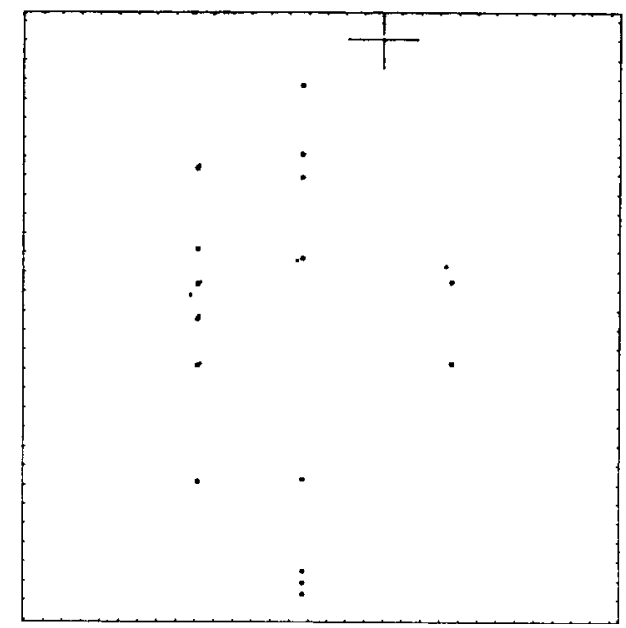

Figure 6

Cartes d'impacts pour la simulation A (après 45 jours). Les graduations du cadre valent 5 cm. Les diamètres des cercles varient suivant les ordres racinaires coupés (les cercles les plus petits correspondent aux racines d'ordre 3). Les croix représentent les projections de la base de la plante sur les plans de coupe.

- A1: coupe horizontale, à $40 \mathrm{~cm}$ de profondeur,

- A2 : coupe verticale, à $25 \mathrm{~cm}$ de la plante, suivant une direction tirée au hasard.

Cross sections of the A pattern (after 45 days). Frame graduations represent $5 \mathrm{~cm}$. Circle diameters depend on the cut root orders (smallest circles are the third-order roots). Crosses represent the projections of the plant base on the section plans.

- Al: horizontal section, at the depth of $40 \mathrm{~cm}$,

- A2: vertical section, at $25 \mathrm{~cm}$ from the plant base.

ment, maille de mesure) des coefficients de variation de la densité racinaire horizontale.

\section{Cartes d'impacts (tabl. 2 et fig. 6)}

Lorsque l'hypothèse précédente d'homogénéité racinaire dans le plan horizontal n'est plus valide, le profil d'enracinement mono-dimensionnel perd beaucoup de sa valeur. L'hétérogénéité de l'enracinement peut résulter de l'isolement des plantes dans certains peuplements (HunT \& NOBEL, 1987) ou des hétérogénéités dans la structure du sol (TARDIEU \& MANICHON, 1986). Ces auteurs montrent sur maïs la variabilité horizontale de la colonisation racinaire liée au compactage du sol lors de travaux agricoles effectués dans de mauvaises conditions.

Dans ces situations, il devient nécessaire de décrire plus en détail la distribution spatiale des racines. L'élément de validation du modèle peut être alors une carte d'impacts racinaires obtenue sur un front de tranchée, horizontal ou vertical (BöHM, 1979).

Un des programmes du logiciel calcule la coupe par un plan horizontal ou vertical choisi par l'utilisateur d'une structure racinaire simulée, ou de plusieurs associées en un petit peuplement (en supposant que les paramètres ont été estimés en peuplement). Il permet ainsi d'obtenir une carte d'impacts théorique (fig. 6) que l'on peut confronter à la réalité. La comparaison nécessite l'utilisation de statistiques adaptées, capables de discriminer des distributions qui vont induire des différences de fonctionnement. Ces statistiques restent à développer en fonction d'objectifs précis.

\section{CONCLUSION}

Le modèle présenté ici constitue avant tout un outil d'analyse et de synthèse pour étudier le système racinaire, aussi bien du point de vue croissance et développement que du point de vue distribution spatiale. Il permet en effet de tester des hypothèses grâce aux sorties calculées qu'elles induisent. Cet outil permet également d'orienter l'expérimentation en précisant par analyse de sensibilité quels sont les paramètres les plus déterminants pour une sortie donnée, et qui méritent donc d'être les mieux étudiés. Comme les structures générées sont stockées sur fichier, il est tout à fait possible de les utiliser pour simuler à la demande différents plans d'échantillonnage (carottes de sol, images d'endoscope) pour optimiser ceux-ci en fonction de ses propres objectifs.

Le programme de simulation est organisé de façon à être une structure d'accueil pour l'intégration de sousmodèles décrivant plus précisément le comportement de chaque racine en fonction de son environnement et de sa position dans la structure tout entière. Il est également envisageable de simuler la mortalité de certains méristèmes.

De cette manière on pourra aborder la modélisation architecturale du système racinaire dans un milieu hétérogène comme le champ, en étudiant notamment les vitesses de croissance, les réactions aux contraintes mécaniques, et le renouvellement des racines. Même si ce travail ne peut être fait sur tous les ordres de racines, la seule connaissance de la disposition de l'ossature du système racinaire est importante si l'on admet que les racines les plus fines se contentent d'exploiter le volume 
enrobant comme une gaine cette charpente (KAHN, 1977).

Cependant, ce modèle ne rend pas compte des corrélations de croissance existant au sein de la plante tout entière, et au sein du système racinaire (BROUWER, 1981), qui peuvent être importantes lorsque le milieu est très hétérogène : cet auteur montre notamment sur maïs qu'une partie du système racinaire peut avoir une croissance nettement augmentée si l'autre partie est en conditions défavorables. Le modèle est toutefois un outil pour décrire précisément ces corrélations de croissance, étape indispensable pour les modéliser.

Le logiciel est écrit en Turbo-Pascal (Borland International) et fonctionne sur toul micro-ordinateur compatible IBM PC. Lo programme de simulation proprement dit occupe environ 16 koctets. Les 4 simulations présentées sur la figure 3 ont demandé entre $20 \mathrm{~s}$ et 90 s sur un compatible PC-AT. Les structures simulées occupent entre 50 et 120 koctets.

Reçu le 23 février 1988. Accepté le 18 uoùt 1988.

\section{RÉFÉRENCES BIBLIOGRAPHIQUES}

Böhm W., 1979. Methods of studying root systems. Ecological Studies, Springer-Verlag, Berlin, $188 \mathrm{p}$.

Brouwer R., 1981. Co-ordination of growth phenomena within a root system of intact maize plants. Plant Soil, 63, 65-72.

Cannon W. A., 1949. A tentative classification of root systems Ecology, 30 (4), 542-548

Charlton W. A., 1983. Patterns of distribution of lateral root primordia. Ann. Bot., 51, 417-427.

De Reffye P., Edelin C., Jeager M., Cabart C., 1986. Simulation de l'architecture des arbres. Naturalia monspeliensa, numéro hors séric Colloque international sur l'Arbre (9-14/09/85), 223-240.

Fitter A. H., 1982. Morphometric analysis of root systems : application of the technique and influence of soil fertility on root system development in two herbaceous species. Plant, Cell Environ., 5, 313-322.

Fitter A. H., 1987. An architectural approach to the comparative ecology of plant root systems. New Physiol., 106, 61-77.

Gerwitz A., Page E. R., 1974. An empirical mathematical model to describe plant root systems. J. appl. Ecol., 11, 773-781.

Hackett C., 1972. A method of applying nutrients locally to roots under controlled conditions, and some morphological effects of locally applied nitrate on the branching of wheat roots. Aust. J. biol. Sci, 25, 1169-1180.

Hackett C., Rose D. A., 1972. A model of the extension and branching of a seminal root of barley, and its use in studying relations between root dimensions. 1. The model. Aust. J. biol. Sci., 25, 669-679.

Huck M. G., Hillel D., 1983. A model of root growth and water uptake accounting for photosynthesis, respiration, transpiration, and soil hydraulics. Adv. Irrig., 2, 273-333.

Hunt E. R., Nobel P. S., 1987. A two-dimensional model for water uptake by desert succulents: implications of root distribution. $A n n$. Bot., 59, 559-569.

Jenik J., 1978. Roots and root systems in tropical trees: morphologic and ecologic aspects. Fourth Cabot symposium. Harvard forest, Petersham, Massachusetts, 1976/04/26-30. In: Tomlinson P. B. Zimmermann M. H. (Eds). Tropical trees as living systems, 14, 323-349. Cambridge University Press.

Kahn F., 1977. Analyse structurale des systèmes racinaires des plantes ligneuses de la forêt tropicale dense humide. Candollea, 32 (2), 321 358.
Klepper B., Belford R. K., Rickman R. W., 1984. Root and shoot development in winter wheat. Agron. J., 76, 117-122.

Kutschera L., 1960. Wurzelatlas mitteleuropäischer Ackernmkrauter und Kulturpflanzen. DLG-Verlags-GmbH, Frankfurt am Main. 574 p.

Lungley D. R., 1973. The growth of root systems. A numerical computer simulation model. Plant Soil, 38, 145-159.

Lyford W. H., 1980. Development of the root system of northern red oak (Quercus mubra L.). Harvard Forest Paper, 21, 15 p. Harvard University, Petersham, Massachusetts.

McCully M. E., 1975. The development of lateral roots. In : Torrey J. G., Clarkson D. T. (Eds). The development and function of roots, 105-124. Academic Press, London.

Page E. R., Gerwitz A., 1974. Mathematical models, based on diffusion equations, to describe root systems of isolated plants, row crops, and swards. Plant Soil, 41, 243-254.

Picard D., Jordan M. O., Trendel R., 1985. Rythme d'apparition des racines primaires du maîs (Zea mays $\mathbf{L}$.). 1 . Etude détaillée pour une variété en un lieu donné. Agronomie, 5(8), 667-676.

Pilet P.-E., 1973. Growth inhibitor from the root cap of Zea mays. Planta, 111, 275-278.

Riedacker A., Belgrand M., 1983. Morphogenèse des systèmes racinaires des semis et boutures de Chêne pédonculć. Plant Soil, $\mathbf{7 1}$, $131-146$.

Riopel J. L., 1966. The distribution of lateral roots in Musa acuminata Gros Michel. Am. J. Bot., 53 (4), 403-407.

Riopel J. L., 1969. Regulation of lateral root positions. Bot. Gaz., 130 (2), 80-83.

Rose D. A., 1983. The description of the growth of root systems. Plant Soil, 75, 405-415.

Tardieu F., Manichon H., 1986. Caractérisation en tant que capteur d'cau de l'enracinement du maïs en parcelle cultivéc. 2. Une méthode d'étude de la répartition verticale el horizontale des racines. Agronomie, 6 (5), 415-425.

Taylor H. M., Klepper B., 1978. Rolc of rooting characteristics. Adv. Agron., 30, 99-128.

Weaver J. E., 1958. Classification of root systems of forbs of grassland and a consideration of their significance. Ecology, 39 (3), 393-401 